\title{
Correction to: VULCAN integrates ChIP-seq with patient-derived co-expression networks to identify GRHL2 as a key co- regulator of ERa at enhancers in breast cancer
}

Andrew N. Holding ${ }^{1,2^{*}+}$ (D, Federico M. Giorgi ${ }^{1,3+}$, Amanda Donnelly ${ }^{1}$, Amy E. Cullen ${ }^{1}$, Sankari Nagarajan ${ }^{1}$, Luke A. Selth ${ }^{4}$ and Florian Markowetz ${ }^{1}$

\section{Correction to: Genome Biol (2019) 20:91 https://doi.org/10.1186/s13059-019-1698-z}

Following publication of the original article [1], the authors reported that Figs. 4 and 5 had mistakenly been transposed. Please find the correct Figs. 4 and 5 below. The original article [1] has been corrected.

\begin{abstract}
Author details
${ }^{1}$ CRUK Cambridge Institute, University of Cambridge, Robinson Way, Cambridge CB2 ORE, UK. ${ }^{2}$ The Alan Turing Institute, 96 Euston Road, Kings Cross, London NW1 2DB, UK. ${ }^{3}$ Department of Pharmacy and Biotechnology, University of Bologna, Via Selmi 3, Bologna, Italy. ${ }^{4}$ Dame Roma Mitchell Cancer Research Laboratories and Freemasons Foundation Centre for Men's Health, Adelaide Medical School, The University of Adelaide, Adelaide, SA, Australia.
\end{abstract}

Received: 7 June 2019 Accepted: 7 June 2019

Published online: 14 June 2019

\section{Reference}

1. Holding AN, et al. VULCAN integrates ChIP-seq with patient-derived co-expression networks to identify GRHL2 as a key co-regulator of ERa at enhancers in breast cancer. Genome Biol. 2019;20:91 https:// doi.org/10.1186/s13059-019-1698-z.

\footnotetext{
* Correspondence: anh25@cam.ac.uk

${ }^{+}$Andrew N. Holding and Federico M. Giorgi contributed equally to this work.

${ }^{1}$ CRUK Cambridge Institute, University of Cambridge, Robinson Way,

Cambridge CB2 ORE, UK

${ }^{2}$ The Alan Turing Institute, 96 Euston Road, Kings Cross, London NW1 2DB,

UK

Full list of author information is available at the end of the article
}

(c) The Author(s). 2019 Open Access This article is distributed under the terms of the Creative Commons Attribution 4.0 International License (http://creativecommons.org/licenses/by/4.0/), which permits unrestricted use, distribution, and reproduction in any medium, provided you give appropriate credit to the original author(s) and the source, provide a link to the Creative Commons license, and indicate if changes were made. The Creative Commons Public Domain Dedication waiver (http://creativecommons.org/publicdomain/zero/1.0/) applies to the data made available in this article, unless otherwise stated. 

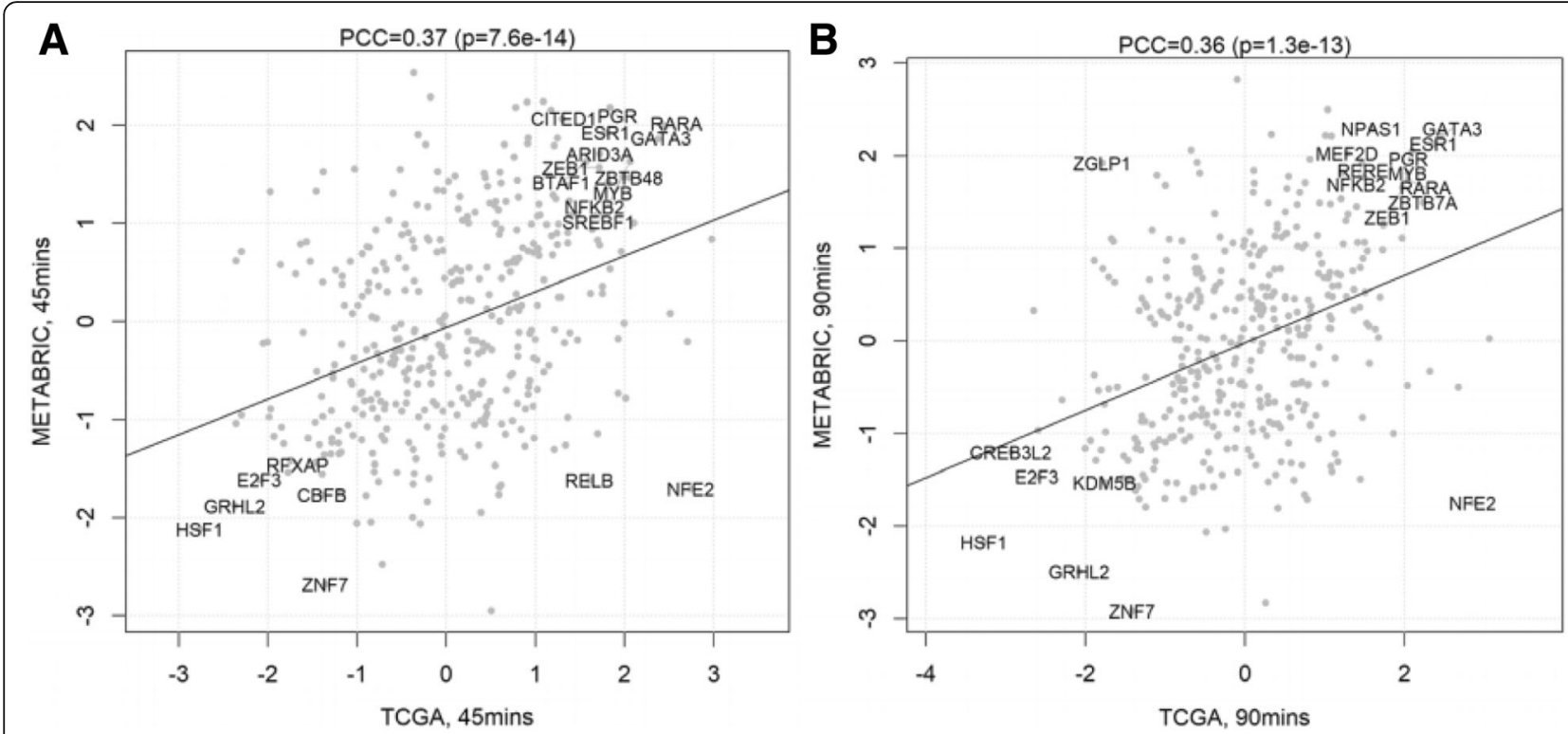

Fig. 4 Global TF activity after estradiol treatment using different network models. XY scatter showing the TF activity as calculated by VULCAN for our differential ChIP-seq analysis of ER binding at $45 \mathrm{~min}(\mathbf{a})$ and at $90 \mathrm{~min}(\mathbf{b})$ after stimulation with $100 \mathrm{nM}$ E2. Comparison of the results calculated using the METABRIC (y-axis) and TCGA ( $x$-axis) networks shows consistent results know ER interactors including PGR, RARA, GATA3, and GRHL2. GRHL2 activity is notably enriched against. The regulon of ER is also consistently enriched in both networks. Pearson's correlation coefficient (PCC) shown along with the significance

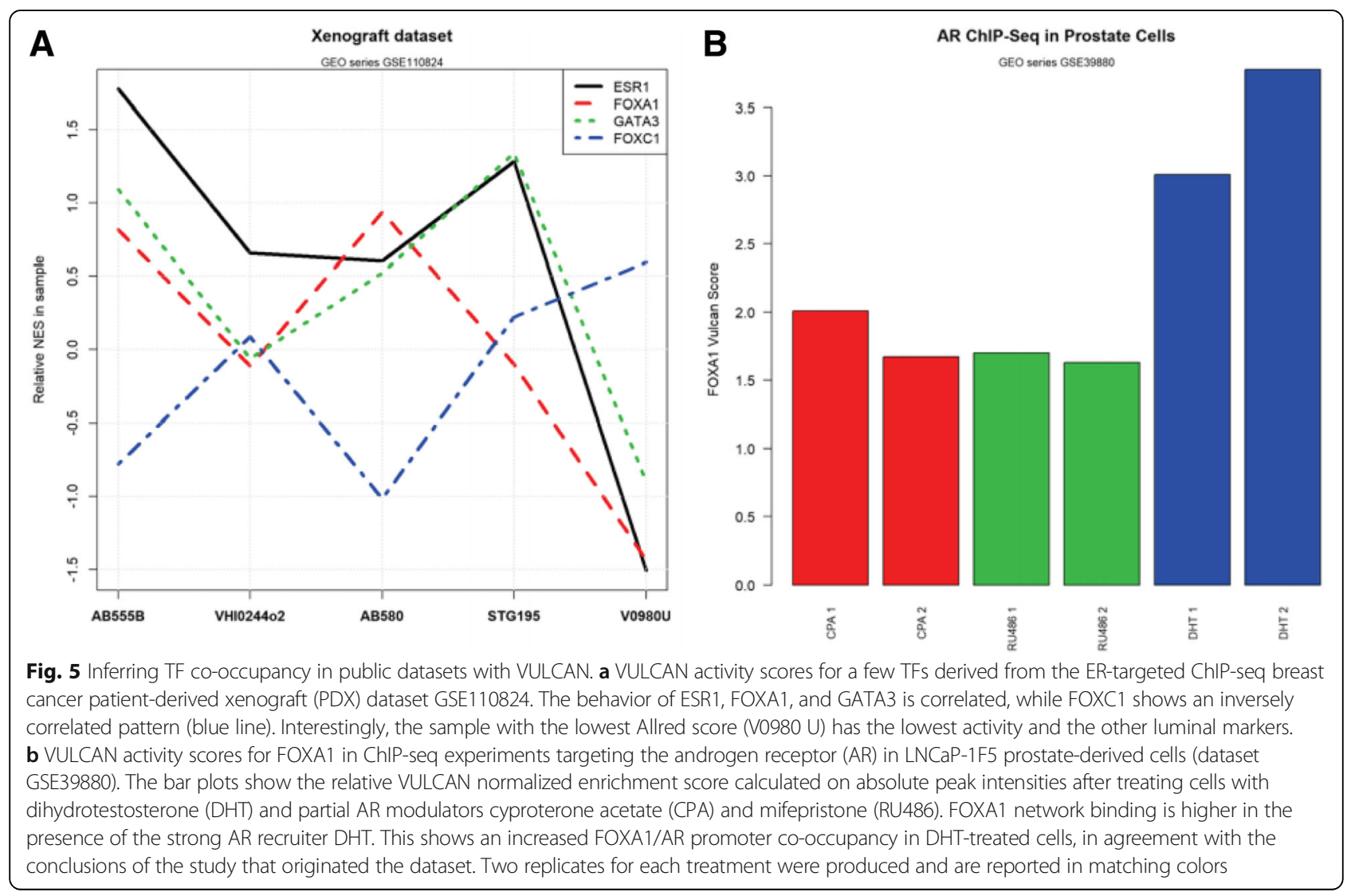

